\title{
Plasmon Modes of Graphene Nanoribbons with Periodic Planar Arrangements
}

\author{
C. Vacacela Gomez, ${ }^{1,2}$ M. Pisarra, ${ }^{1,3}$ M. Gravina, ${ }^{1,2}$ J. M. Pitarke, ${ }^{4,5}$ and A. Sindona ${ }^{1,2, *}$ \\ ${ }^{1}$ Dipartimento di Fisica, Università della Calabria, Via P. Bucci, Cubo 30C, I-87036 Rende (CS), Italy \\ ${ }^{2} I N F N$, Sezione LNF, Gruppo Collegato di Cosenza, Cubo 31C, I-87036 Rende (CS), Italy \\ ${ }^{3}$ Departamento de Química, Universidad Autónoma de Madrid, Calle Francisco Tomás y Valiente 7 (Módulo 13), \\ E-28049 Madrid, Spain \\ ${ }^{4}$ CIC nanoGUNE, Tolosa Hiribidea 76, E-20018 Donostia-San Sebastian, Basque Country, Spain \\ ${ }^{5}$ Materia Kondentsatuaren Fisika Saila, DIPC, and Centro Fisica Materiales CSIC-UPV/EHU, 644 Posta Kutxatila, \\ E-48080 Bilbo, Basque Country, Spain
}

(Received 30 January 2016; revised manuscript received 28 May 2016; published 7 September 2016)

\begin{abstract}
Among their amazing properties, graphene and related low-dimensional materials show quantized charge-density fluctuations-known as plasmons-when exposed to photons or electrons of suitable energies. Graphene nanoribbons offer an enhanced tunability of these resonant modes, due to their geometrically controllable band gaps. The formidable effort made over recent years in developing graphene-based technologies is however weakened by a lack of predictive modeling approaches that draw upon available $a b$ initio methods. An example of such a framework is presented here, focusing on narrowwidth graphene nanoribbons, organized in periodic planar arrays. Time-dependent density-functional calculations reveal unprecedented plasmon modes of different nature at visible to infrared energies. Specifically, semimetallic (zigzag) nanoribbons display an intraband plasmon following the energymomentum dispersion of a two-dimensional electron gas. Semiconducting (armchair) nanoribbons are instead characterized by two distinct intraband and interband plasmons, whose fascinating interplay is extremely responsive to either injection of charge carriers or increase in electronic temperature. These oscillations share some common trends with recent nanoinfrared imaging of confined edge and surface plasmon modes detected in graphene nanoribbons of 100-500 nm width.
\end{abstract}

DOI: 10.1103/PhysRevLett.117.116801

Plasmons are quantized oscillations of the valence electron density in metals, metal-dielectric interfaces, and nanostructures, being usually excited by light or electron-beam radiation. Plasmon-related technologies are expected to receive a burst from nanocarbon architectures [1-10], due to one of the fascinating features of monolayer graphene (MG) [11], i.e., its extrinsic plasmon modes at terahertz $(\mathrm{THz})$ frequencies [12-20]. These show much stronger confinement, larger tunability, and lower losses [21] compared to conventional plasmonic materials, such as silver or gold. Nowadays plasmons are launched, controlled, manipulated, and detected in a variety of graphene-related materials and heterostructures, which suggests that graphene-based plasmonic devices are becoming closer to reality, with the potential to operate on the "THz gap," forbidden by either classical electronics or photonics $[4,22,23]$. Plasmons with widely tunable frequencies have been observed in graphene nanoribbons (GNRs)-from the nano- to microrange in width $[12,14,24,25]$. On the theoretical side, density functional and tight-binding (TB) approaches have explored the electronic structure of zigzag and armchair GNRs, with particular attention to the band-gap values of the intrinsic systems, being a major control factor of their plasmonic properties [26-30]. Far fewer studies have been focused on plasmon resonances in GNRs using either a semiclassical electromagnetic picture [31] or a TB scheme [5,32,33], and specializing to $\mathrm{THz}$ frequencies. A comprehensive characterization of the dielectric properties of such systems is, however, lacking.

Here, we provide an $a b$ initio study of plasmon excitations in regular planar arrays of GNRs, sorting a wide range of frequencies, from the lower $\mathrm{THz}$ to extreme ultraviolet (UV). We use time-dependent (TD) density functional theory (DFT) in the random-phase approximation (RPA), emphasizing the 4ZGNR and 5AGNR geometries, which are respectively characterized by four zigzag chains [Fig. 1(a)] and five dimer lines [Fig. 1(c)] across the GNR width [26]. The dangling bonds of each GNR array are passivated by hydrogen atoms on both sides, with the $\mathrm{C}-\mathrm{C}$ and $\mathrm{C}-\mathrm{H}$ bond lengths being fixed to their nominal values, which differ by less than $1 \%$ from the corresponding geometrically optimized values (Figs. S1 and S2 in [34]). The GNR arrays are separated by an in-plane vacuum width of $15 \AA$ [Figs. 1(a) and 1(c)], while periodic boundary conditions are used for the direction parallel to the GNR axis, which mimics a situation of long suspended ribbons with fixed edges on the far ends. The equilibrium electronic structures of the systems (Sec. I in [34]) are computed using the local-density approximation (LDA [35]) with normconserving pseudopotentials [36] and the plane-wave (PW) basis $[37,38]$. The three-dimensional periodicity required by PW DFT is generated by replicating the GNR arrays over an out-of-plane distance $L$ of $15 \AA$, which ensures negligible 


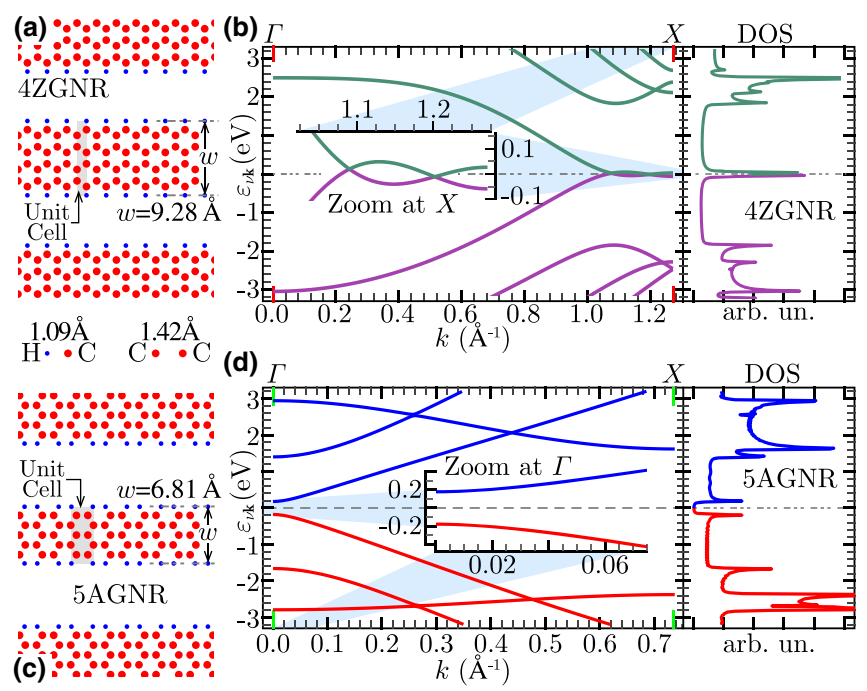

FIG. 1. Geometry [(a) and (c)], band energies (zoomed at $E_{F}=0$ ), and DOS [(b) and (d)] for 4ZGNR [(a) and (b)] and $5 \mathrm{AGNR}[(\mathrm{c})$ and $(\mathrm{d})]$.

overlap (but not negligible interaction) of charge density between the replicas.

Accordingly, the different geometry of the assemblies [Figs. 1(a) and 1(c)] produces electronically distinct band dispersions and densities of states (DOS). 4ZGNR [Fig. 1(b)] appears as a semimetal with the valence and conduction bands overlapping close to the $X$ point. The quasiflat dispersions near the intrinsic Fermi-level $E_{F}$ give rise to strong peaks in the DOS, as opposite to MG where the linear dispersing valence and conduction levels yield a vanishing DOS at $E_{F}$. 5AGNR [Fig. 1(d)] is a semiconductor with the valence and conduction electrons having paraboliclike dispersions around a small gap of $\sim 0.36 \mathrm{eV}$ at the $\Gamma$ point that result in two peaks in the DOS. It should be noted that local spin density calculations suggest the opening of a band gap larger than $0.1 \mathrm{eV}$ in ZGNRs [26-28]. Additionally, $G W$ approaches predict larger band gaps in both ZGNRs and AGNRs by roughly $1 \mathrm{eV}$ with respect to local density calculations [27]. Nonetheless, band-gap values of the same order of the LDA band gap of 5AGNR have been measured for some GNRs as wide as about $20 \mathrm{~nm}$ grown on $\mathrm{Au}(111)$ [30]. Thus, the application of an RPA scheme to the LDA band structure of 5AGNR can be of help in interpreting plasmon measurements on currently synthesized GNR structures [25]. Complementarily, the LDA analysis of a virtually gapless GNR, i.e., 4ZGNR, is particularly instructive to emphasizing the different role played by doping.

The starting point of our TDDFT approach is the densitydensity response function of noninteracting electrons in the GNRs, as given by the Adler-Wiser formula $[39,40]$

$$
\chi_{\mathbf{G G}^{\prime}}^{0}=\frac{2}{\Omega} \sum_{\mathbf{k}, \nu, \nu^{\prime}} \frac{\left(f_{\nu \mathbf{k}}-f_{\nu^{\prime} \mathbf{k}+\mathbf{q}}\right) \rho_{\nu \nu^{\prime}}^{\mathbf{k q}}(\mathbf{G}) \rho_{\nu \nu^{\prime}}^{\mathbf{k q}}\left(\mathbf{G}^{\prime}\right)^{*}}{\omega+\varepsilon_{\nu \mathbf{k}}-\varepsilon_{\nu^{\prime} \mathbf{k}+\mathbf{q}}+i \eta},
$$

which is a corollary of the Kubo formula for a periodic system with associated reciprocal lattice and Brilloiun Zone (BZ) [41]. Hartree atomic units are used throughout this Letter, unless otherwise stated.

In Eq. (1) the electron energies $\varepsilon_{\nu \mathbf{k}}$ and states $|\nu \mathbf{k}\rangle$ are indexed by the band number $\nu$ and the wave vector $\mathbf{k}$ in the first BZ. These are taken to be the Kohn-Sham (KS) eigensystems of our PW-DFT approach, leading to the electronic structure of Figs. 1(b) and 1(d). The KS wave functions, normalized to unity in the volume $\Omega$, are expressed as linear combinations of PWs that depend on the reciprocal-lattice vectors $\mathbf{G}$ associated to the replicated GNR lattices (Secs. I and II in [34]). The correlation matrix elements are given by $\rho_{\nu \nu^{\prime}}^{\mathbf{k q}}(\mathbf{G})=\left\langle\nu \mathbf{k}\left|e^{-i(\mathbf{q}+\mathbf{G}) \cdot \mathbf{r}}\right| \nu^{\prime} \mathbf{k}+\mathbf{q}\right\rangle$. The population of single-particle levels is established by the Fermi-Dirac distribution $f_{\nu \mathbf{k}}$, which we evaluate by sampling temperatures from 300 to $900 \mathrm{~K}$. The factor of 2 accounts for the spin degeneracy, while $\eta$ is a small (positive) lifetime broadening parameter [42].

Polarization effects are activated by a test electron or photon with incident energy $\omega$ and in-plane momentum q that weakly perturbs our systems. These are described by the density-density response function of interacting electrons, which can be obtained in the TDDFT framework as $\chi_{\mathbf{G G}^{\prime}}=\chi_{\mathbf{G G}^{\prime}}^{0}+\left(\chi^{0} v \chi\right)_{\mathbf{G G}^{\prime}}[43,44]$.

In the RPA, one neglects short-range exchangecorrelation effects by simply replacing the unknown $v$ by the bare Coulomb terms, $v_{\mathbf{G G}^{\prime}}^{0}=4 \pi \delta_{\mathbf{G G}^{\prime}} /|\mathbf{q}+\mathbf{G}|^{2}$. A serious drawback stems from the long-range character of the Coulomb potential, which allows non-negligible interactions between repeated planar arrays even at large distances. To cut off this unwanted phenomenon, we replace $v_{\mathbf{G G}^{\prime}}^{0}$ by the truncated Fourier integral [18,19,45-47],

$$
v_{\mathbf{G G}^{\prime}}=\int_{-L / 2}^{L / 2} d z \int_{-L / 2}^{L / 2} d z^{\prime} e^{i G z} \bar{v}_{\mathbf{g g}^{\prime}}^{0}\left(z, z^{\prime}\right) e^{-i G^{\prime} z^{\prime}}
$$

where $\bar{v}_{\mathbf{g g}}^{0}$ is the Fourier transform of $v_{\mathbf{G G}^{\prime}}^{0}$ along the out-ofplane axis, while $\mathbf{g}$ and $G$ denote the in-plane and out-ofplane components of $\mathbf{G}$.

Within linear response theory, the inelastic cross section corresponding to a process where the external perturbation creates an excitation of energy $\omega$ and wave vector $\mathbf{q}+\mathbf{G}$ is related to the diagonal elements of the inverse dielectric matrix: $\left(\epsilon^{-1}\right)_{\mathbf{G G}^{\prime}}=\delta_{\mathbf{G G}^{\prime}}+(v \chi)_{\mathbf{G G}^{\prime}}$. Collective excitations (plasmons) are dictated by the zeros in the real part of the macroscopic dielectric function (permittivity): $\epsilon^{M}=1 /\left(\epsilon^{-1}\right)_{\mathbf{0 0}}$. The so-called energy-loss (EL) function is proportional to the imaginary part of the inverse permittivity: $E_{\mathrm{LOSS}}=-\operatorname{Im}\left[\left(\epsilon^{-1}\right)_{\mathbf{0 0}}\right]$. Nonlocal field effects are included in $E_{\mathrm{LOSS}}$ through the off-diagonal elements of $\chi_{\mathbf{G G}^{\prime}}$ [48].

The peculiar electronic structure of 4ZGNR [Fig. 1(b)] and 5AGNR [Fig. 1(d)], as compared to the well-known 
$E_{\mathrm{LOSS}}$ at $300 \mathrm{~K}$
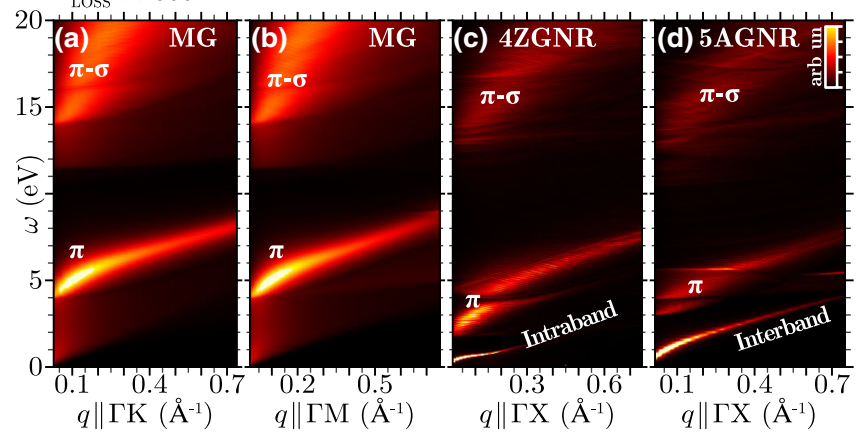

FIG. 2. $E_{\mathrm{LOSS}}$ vs $\omega<20 \mathrm{eV}$ and $q<0.8 \AA^{-1}$ for intrinsic $\mathrm{MG}$ [(a) and (b)], 4ZGNR (c), and 5AGNR (d).

band dispersion of MG, is reflected in the EL spectra of the intrinsic systems shown in Fig. 2. Undoped 4ZGNR and 5AGNR have two high-energy excitations (for $\omega>3 \mathrm{eV}$ ) that follow one-electron transitions connecting the $\mathbf{k}$ points with high DOS in the $\pi-\pi^{*}, \sigma-\pi^{*}$, and $\pi-\sigma^{*}$ bands. These are counterparts to the $\pi$ and $\sigma-\pi$ interband plasmons observed in intrinsic MG [Figs. 2(a) and 2(b)], few-layer graphene, and graphite [49-52]. Specifically, the $\pi$ and $\pi-\sigma$ structures of the GNRs exhibit a discontinuous dispersion vs $\mathbf{q}$ and $\omega$, as they are split into more branches [Figs. 2(c), 2(d) and Figs. S3(c), S3(d) in [34]]. This is due to the finite width of the GNRs in the periodic array, generating several one-dimensional bands of $\pi$ and $\sigma$ character.

The number and dispersion of these bands is also strongly influenced by the GNR width, chirality, and inplane distance: the wider the ribbon the more regular the high-energy interband peaks, which approach the $\pi$ and $\sigma-\pi$ peaks of MG as the ribbon width tends to infinity [Figs. 2(a), 2(b) and Figs. S3(a), S3(b) in [34]]. Then, the main designing "ingredients" of the GNR arrays may be finely tuned to reach a specific energy for the $\pi$ and $\sigma-\pi$ excitations, which in turn may be used to change the response of a GNR-based device working in the visible (VIS) to UV regime. The low-energy ends of the spectra (for $\omega<3 \mathrm{eV}$ ) exhibit an extra peak in both metallic and semiconducting GNRs, which is strictly absent in MG at the absolute zero. These structures are more detailed in Figs. 3(a), 3(e), 4(a) and 4(e) below. The large DOS value close to $E_{F}$ in 4ZGNR [Fig. 1(b)] yields a concentration of $n^{*}=3.96 \times 10^{12} \mathrm{~cm}^{-2}$ conduction electrons, which allows the appearance of an intraband plasmon where the charge carriers located on each ribbon of the array oscillate as a single two-dimensional gas [Figs. 2(c), 3(a) and Figs. S3(c), S4(a) in [34]]. This observation is confirmed by the typical square root dispersion of two-dimensional plasmons [53] for low $q$ [Fig. S4(d) in [34]] that has been mostly observed in extrinsic MG [17], which even in the intrinsic case allows for a weak intraband mode at room temperature assisted by the conduction-electron concentration $n^{*}=1.15 \times 10^{11} \mathrm{~cm}^{-2}$.
$4 \mathrm{ZGNR} \rightarrow E_{\mathrm{LOSS}}$ at $300 \mathrm{~K}$
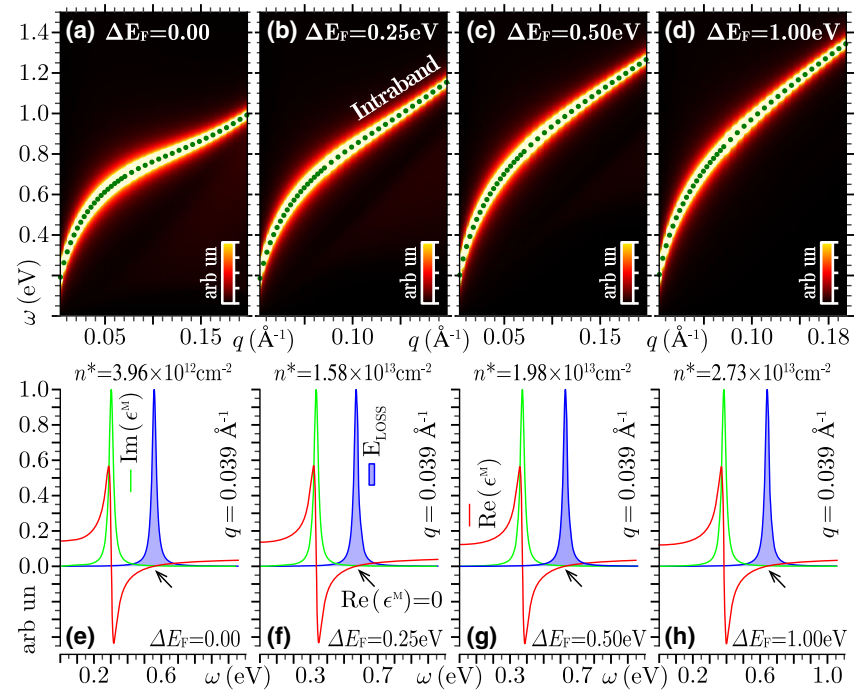

FIG. 3. EL spectrum and complex permittivity of intrinsic and extrinsic 4ZGNRs at room temperature. [(a)-(d)] $E_{\text {LOSS }}$ vs $\omega<1.5 \mathrm{eV}$ and $q<0.11 \AA^{-1}$. [(e)-(h)] $\operatorname{Re}\left(\epsilon^{M}\right), \operatorname{Im}\left(\epsilon^{M}\right)$ and $E_{\text {LOSS }}$ vs $\omega<1.5 \mathrm{eV}$ at $q=0.039 \AA^{-1}$. In (a)-(d) the same color code or intensity scale as in Fig. 2 is used, with the green dots denoting the $(\omega, q)$ dispersion of the intraband plasmon.

On the other hand, the energy gap at $E_{F}$ in 5AGNR [Fig. 1(d)] yields a negligibly small intraband mode due to the tiny concentration of conduction electrons at room temperature $\left(n^{*}=8.70 \times 10^{8} \mathrm{~cm}^{-2}\right)$. The latter is not detectable in Figs. 2(d) and 4(a) but can be, in principle, characterized (Sec. III in [34]). In contrast, an interband

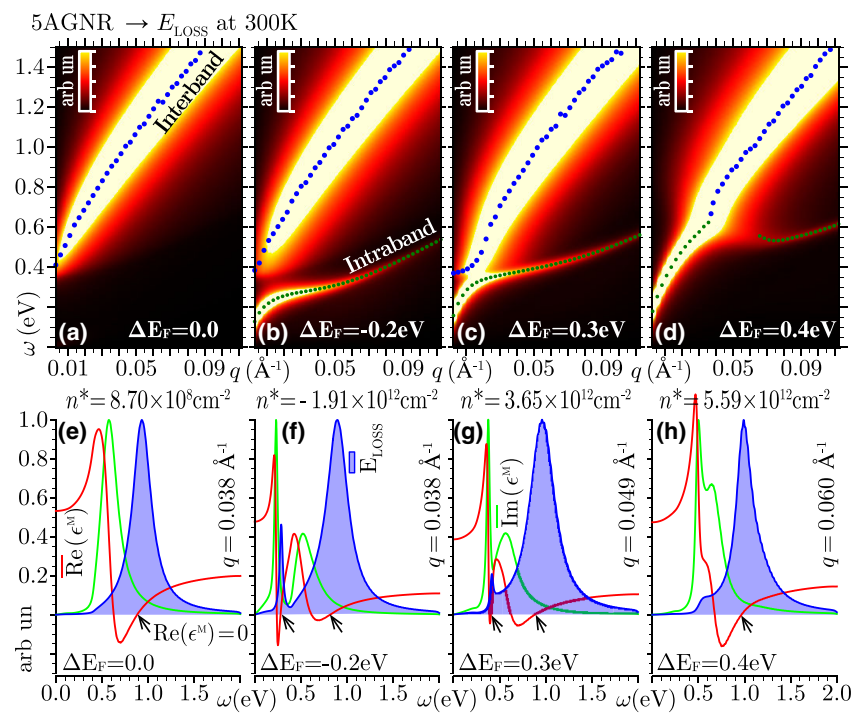

FIG. 4. Dielectric response and plasmon dispersions of intrinsic and extrinsic 5AGNRs at $T=300 \mathrm{~K}$. [(a)-(d)] $E_{\text {LOSS }}$ vs $\omega<$ $1.5 \mathrm{eV}$ and $q<0.11 \AA^{-1}$ with the same intensity scale as Figs. 2 and $3(\mathrm{a})-3(\mathrm{~d})$. The blue and green dots mark the interband and intraband plasmon $(\omega, q)$ dispersions, respectively. 
two-dimensional plasmon is clearly recorded in the lowenergy spectrum of 5AGNR, as testified by the intense signal in Figs. 2(d), 4(a) and 4(d); this corresponds to a collective mode that is triggered by transitions between the valence and conduction DOS peaks at $\Gamma$ [Fig. 1(d)]. The collective nature of the newly detected modes in 4ZGNR and 5AGNR is proved in Figs. 3(e) and 4(e), respectively, where we see that each excitation peak in the EL spectrum corresponds to a zero in the real permittivity $\operatorname{Re}\left(\epsilon^{M}\right)$, at a frequency where the imaginary permittivity $\operatorname{Im}\left(\epsilon^{M}\right)$ is small. Moreover, as is the case for the high-energy $\pi$ excitations, these low-energy modes arise from transitions involving the $\pi$ and $\pi^{*}$ bands, which means that their intensities and energy-momentum dispersions can be modulated according to external factors that change the band levels, such as the already mentioned ribbon width, in-plane distance, and chirality.

Let us now see how the dielectric properties of the GNR arrays behave with injecting or ejecting electrons by doping or gating. Extrinsic systems are simulated here by slightly changing the level populations in Eq. (1), in such a way that band dispersions and single-particle KS orbitals are negligibly altered by the applied variations of the $f_{\nu \mathbf{k}}$ factors. For doping levels $\Delta E_{F}$ not larger than $\sim 1 \mathrm{eV}$ the highenergy end $(\omega>3 \mathrm{eV})$ of our EL spectra is practically unaffected. On the contrary, unprecedented new features are recorded at the low-energy end $(\omega<3 \mathrm{eV})$.

In Figs. 3(a)-3(d) [and Figs. S4(a)-S4(c) in [34]] we show the low- $\omega$ and low-q region of the EL spectrum of 4ZGNR arrays, zooming on the undoped case and analyzing three positive doping levels, whose charge-carrier concentrations are consistent with the measurements of Ref. [54]. We observe a single dispersive structure, the intraband plasmon, which is a genuine collective mode, with the EL peak corresponding to a zero in $\operatorname{Re}\left(\epsilon^{M}\right)$ and a small value of $\operatorname{Im}\left(\epsilon^{M}\right)$ [Figs. 3(e)-3(h)]. We also notice minor differences in the four EL spectra, with the plasmon energy slightly increasing with increasing $\Delta E_{F}$ up to $1.0 \mathrm{eV}$ [Fig. S4(d) in [34]].

More interesting features are observed in doped 5AGNR arrays, whose low- $\omega$ and low-q response is shown in Fig. 4. In the undoped case, a single dispersive peak is detected that represents an interband plasmon, which follows coherent one-electron transitions between valence and conduction states [Figs. 4(a), 4(d) and Fig. S5(c) in [34]]. When a small doping is introduced $\left(\Delta E_{F}=-0.2\right.$, $0.3 \mathrm{eV})$ the conduction-electron or valence-hole concentration bursts from $\pm 10^{9}$ to $\pm 10^{12} \mathrm{~cm}^{-2}$, a value reported in previous experiments [55]. Then, another dispersive peak appears due to a clearly resolved intraband plasmon [Figs. 4(b), 4(c), 4(f), 4(g) and Figs. S5(b), S5(e) in [34]].

For these low wave vectors $\left(q<0.02 \AA^{-1}\right)$, the intraband mode is the most intense contribution, while the interband plasmon is depressed because the doping partially fills the conduction band near $\Gamma$, thus inhibiting quasivertical $(q \rightarrow 0, \omega)$ interband transitions. In the $0.02<q<0.06 \AA^{-1}$ region, both the intraband and interband plasmons coexist. At larger values of $q$, the interband plasmon becomes the most intense peak while the intraband plasmon is strongly damped.

A slightly larger value of the doping $\left(\Delta E_{F}=0.4 \mathrm{eV}\right)$ leads to an even more intriguing situation: the single dispersive peak visible in Figs. 4(d) and 4(h) has a double nature, as testified by the kink in peak dispersion and the abrupt decrease in intensity (increase in width) found at $q \sim 0.05 \AA^{-1}$ [Fig. S5(f) in [34]]. Indeed, interband transitions between the high-DOS points of Fig. 1(d) for $q<$ $0.04 \AA^{-1}$ are strongly quenched by electron population of conduction levels; thus, the intense peak showing the $\sqrt{q}$ dispersion is mostly originated by the intraband plasmon. Conversely, for $q>0.04 \AA^{-1}$ the intraband plasmon enters a region where it is damped by interband transitions; as a result, most of the spectral weight is concentrated on the interband plasmon, while the overdamped intraband plasmon only appears as a faint peak. Another remarkable effect is the high sensitivity of the intraband plasmon to the type of doping; opposite doping levels, such as the $\Delta E_{F}=$ \pm 0.2 and $\Delta E_{F}= \pm 0.3$ values of Fig. S5(h) in [34], produce significantly different charge-carrier concentrations and plasmon dispersion curves for energies larger than $0.1 \mathrm{eV}$ and transferred momenta above $0.02 \AA^{-1}$. Such a sensitivity is ascribed to the slight asymmetry of the valence and conduction electron levels of 5AGNR close to the band gap [inset in Fig. 1(d)].

On the other hand, the interband plasmon is much less influenced by the doping type, because the valence and conduction DOS peaks have similar intensities [Fig. S5(h) in [34]]. The interplay of the two modes is also modulated by changes in the incident momentum direction, relative to the ribbon axis, due to the tensor character of the GNR dielectric response (Fig. S6 in [34]). In the lower THz region, interband transitions are forbidden by the band gap. The intraband plasmon follows an $\omega$-vs- $q$ dispersion that is consistent with the semiphenomenological relation of Ref. [31], and appears at the same scale as the low- $q$ dispersions of Ref. [33], computed from the TB approximation where 5AGNR is virtually gapless [Fig. S7(a) in [34]]. Then, the TDDFT response of narrow-width GNRs has the correct $q \rightarrow 0, \omega \rightarrow 0$ limiting behavior predicted by (non- $a b$ initio) approaches on larger GNR structures, currently available for experiments.

As a final remark we observe that in semiconducting GNRs, due to their small band gap, the temperature plays a role in dictating the populations of the levels close to $E_{F}$ (Sec. IV in [34]). Accordingly, the intraband plasmon mode can be triggered by working at temperatures larger than $\sim 500 \mathrm{~K}$, which may have a crucial role in relation with the power consumption of nanodevices. Charge-carrier concentrations generated by temperature increase are nevertheless 
much smaller than those obtained with doping or gating. For this reason, no particular interference is recorded between intraband and interband plasmon modes (Fig. S8 in [34]).

In summary, we have discussed the dielectric properties and plasmon dispersions in planar GNR arrays scrutinizing the excitation energy regime going from the THz to the UV scale by an ab initio strategy based on TDDFT + RPA.

On the $\mathrm{THz}$ regime, we have detected new collective modes of different nature. Semimetallic GNRs display an intraband two-dimensional plasmon with large intensity relative to the high-energy plasmons even in the intrinsic case. Semiconducting GNRs experience a fascinating interplay of intraband and interband collective modes, whose relative intensities and dispersions are strongly influenced by the actual occupation of single-particle levels near the Fermi energy.

Some recent calculations have reported the existence of two extrinsic plasmons in MG and bilayer graphene (BLG) [17-20]. In particular, the plasmon coupling in BLG [20] shares some common features with 5AGNR at similar doping conditions; i.e., one of the two modes disperses like $q^{1 / 2}$ and the other is quasivertical.

Indeed, a first experimental evidence of an edge (interband) plasmon superimposed to a conventional (intraband) plasmon has been given in patterned GNRs grown on $\mathrm{Al}_{2} \mathrm{O}_{3}$ [25]. The two modes are well resolved in space on GNR samples of $480 \mathrm{~nm}$ width, at a working frequency of $\sim 0.15 \mathrm{eV}$ and a doping level of $\sim 0.3 \mathrm{eV}$. In our narrowwidth GNR, the interband and intraband features are resolved in momentum space, only.

Our calculations demonstrate that it is possible to construct new materials with plasmonic resonances that are tunable to suit a specific demand in both the VIS-UV and $\mathrm{THz}$ regimes, by means of chemical doping, electronic gating, and also a careful choice of the geometry. These findings if confirmed by further experiments will widen the perspectives on applications of GNR arrays for the engineering of nanophotonic and nanoelectronic devices.

C. V. G. acknowledges the financial support of "Secretaria Nacional de Educación Superior, Ciencia, Tecnología e Innovación" (SENESCYT-ECUADOR). All authors thank Dr. V. M. Silkin from the University of the Basque Country for his invaluable support in developing the TDDFT code.

*antonello.sindona@fis.unical.it

[1] Y.-M. Lin, K. A. Jenkins, A. Valdes-Garcia, J. P. Small, D. B. Farmer, and P. Avouris, Nano Lett. 9, 422 (2009).

[2] M. V. Shuba, G. Y. Slepyan, S. A. Maksimenko, C. Thomsen, and A. Lakhtakia, Phys. Rev. B 79, 155403 (2009).

[3] Q. Bao and K. P. Loh, ACS Nano 6, 3677 (2012).

[4] B. Sensale-Rodriguez, R. Yan, M. M. Kelly, T. Fang, K. Tahy, W. S. Hwang, D. Jena, L. Liu, and H. G. Xing, Nat. Commun. 3, 780 (2012).
[5] S. Thongrattanasiri, A. Manjavacas, and F. J. G. de Abajo, ACS Nano 6, 1766 (2012).

[6] Yu. V. Bludov, A. Ferreira, N. M. R. Peres, and M. I. Vasilevskiy, Int. J. Mod. Phys. B27, 1341001 (2013).

[7] T. Low and P. Avouris, ACS Nano 8, 1086 (2014).

[8] F. J. Garcia de Abajo, ACS Photonics 1, 135 (2014).

[9] C. Brun, T. C. Wei, P. Franck, Y. C. Chong, L. Congxiang, C. W. Leong, D. Tan, T. B. Kang, P. Coquet, and D. Baillargeat, IEEE Trans. Terahertz Sci. Technol. 5, 383 (2015).

[10] Z. Fei, E. G. Iwinski, G. X. Ni, L. M. Zhang, W. Bao, A. S. Rodin, Y. Lee, M. Wagner, M. K. Liu, S. Dai, M. D. Goldflam, M. Thiemens, F. Keilmann, C. N. Lau, A. H. Castro-Neto, M. M. Fogler, and D. N. Basov, Nano Lett. 15, 4973 (2015).

[11] A. C. Neto, F. Guinea, N. Peres, K. S. Novoselov, and A. K. Geim, Rev. Mod. Phys. 81, 109 (2009).

[12] L. Ju et al., Nat. Nanotechnol. 6, 630 (2011).

[13] W. Zhou, J. Lee, J. Nanda, S. T. Pantelides, S. J. Pennycook, and J.-C. Idrobo, Nat. Nanotechnol. 7, 161 (2012).

[14] H. Yan, T. Low, W. Zhu, Y. Wu, M. Freitag, X. Li, F. Guinea, P. Avouris, and F. Xia, Nat. Photonics 7, 394 (2013).

[15] S. C. Liou, C.-S. Shie, C. H. Chen, R. Breitwieser, W. W. Pai, G. Y. Guo, and M.-W. Chu, Phys. Rev. 91B, 045418 (2015).

[16] A. Cupolillo, A. Politano, N. Ligato, D. C. Perez, G. Chiarello, and L. Caputi, Surf. Sci. 634, 76 (2015).

[17] M. Pisarra, A. Sindona, P. Riccardi, V. M. Silkin, and J. M. Pitarke, New J. Phys. 16, 083003 (2014).

[18] M. Pisarra, A. Sindona, M. Gravina, V. M. Silkin, and J. M. Pitarke, Phys. Rev. B 93, 035440 (2016).

[19] A. Sindona, M. Pisarra, D. Mencarelli, L. Pierantoni, and S. Bellucci, Fundamental and Applied Nanoelectromagnetics, edited by A. Maffucci and S. A. Maksimenko (Springer, New York, 2016), Chap. 7, pp. 125-144.

[20] T. Low, F. Guinea, H. Yan, F. Xia, and P. Avouris, Phys. Rev. Lett. 112, 116801 (2014).

[21] J. Christensen, A. Manjavacas, S. Thongrattanasiri, F. H. Koppens, and F. J. García de Abajo, ACS Nano 6, 431 (2012).

[22] A. Woessner et al., Nat. Mater. 14, 421 (2015).

[23] J. Tong, M. Muthee, S.-Y. Chen, S. K. Yngvesson, and J. Yan, Nano Lett. 15, 5295 (2015).

[24] A. Y. Nikitin, F. Guinea, F. J. Garcia-Vidal, and L. MartinMoreno, Phys. Rev. B 85, 081405 (2012).

[25] Z. Fei et al., Nano Lett. 15, 8271 (2015).

[26] Y.-W. Son, M. L. Cohen, and S. G. Louie, Phys. Rev. Lett. 97, 216803 (2006).

[27] L. Yang, C.-H. Park, Y.-W. Son, M. L. Cohen, and S. G. Louie, Phys. Rev. Lett. 99, 186801 (2007).

[28] S. M. M. Dubois, Z. Zanolli, X. Declerck, and J. C. Charlier, Eur. Phys. J. B 72, 1 (2009).

[29] E.-j. Kan, Z. Li, J. Yang, and J. Hou, J. Am. Chem. Soc. 130, 4224 (2008).

[30] C. Tao et al., Nat. Phys. 7, 616 (2011).

[31] V. V. Popov, T. Y. Bagaeva, T. Otsuji, and V. Ryzhii, Phys. Rev. B 81, 073404 (2010).

[32] L. Brey and H. A. Fertig, Phys. Rev. B 73, 235411 (2006). 
[33] D. R. Andersen and H. Raza, Phys. Rev. B 85, 075425 (2012).

[34] See Supplemental Material at http://link.aps.org/ supplemental/10.1103/PhysRevLett.117.116801 for the following: DFT calculations and geometry optimizations (Sec. I); the TDDFT scheme and intrinsic GNRs (Sec. II); doped GNRs, and interband and intraband plasmon dispersions (Sec. III); and temperature effects (Sec. IV).

[35] J. P. Perdew and A. Zunger, Phys. Rev. B 23, 5048 (1981).

[36] N. Troullier and J. L. Martins, Phys. Rev. B 43, 1993 (1991).

[37] X. Gonze et al., Comput. Phys. Commun. 180, 2582 (2009).

[38] H. J. Monkhorst and J. D. Pack, Phys. Rev. B 13, 5188 (1976).

[39] S. L. Adler, Phys. Rev. 126, 413 (1962).

[40] N. Wiser, Phys. Rev. 129, 62 (1963).

[41] R. Kubo, J. Phys. Soc. Jpn. 12, 570 (1957).

[42] V. M. Silkin, E. V. Chulkov, and P. M. Echenique, Phys. Rev. B 68, 205106 (2003).

[43] M. Petersilka, U. J. Gossmann, and E. K. U. Gross, Phys. Rev. Lett. 76, 1212 (1996).

[44] G. Onida, L. Reining, and A. Rubio, Rev. Mod. Phys. 74, 601 (2002).

[45] V. Despoja, K. Dekanić, M. Šunjić, and L. Marušić, Phys. Rev. B 86, 165419 (2012).
[46] V. Despoja, D. Novko, K. Dekanić, M. Šunjić, and L. Marušić, Phys. Rev. B 87, 075447 (2013).

[47] D. Novko, V. Despoja, and M. Šunjić, Phys. Rev. B 91, 195407 (2015).

[48] C. Kramberger, R. Hambach, C. Giorgetti, M. H. Rümmeli, M. Knupfer, J. Fink, B. Büchner, Lucia Reining, E. Einarsson, S. Maruyama, F. Sottile, K. Hannewald, V. Olevano, A. G. Marinopoulos, and T. Pichler, Phys. Rev. Lett. 100, 196803 (2008).

[49] T. Eberlein, U. Bangert, R. R. Nair, R. Jones, M. Gass, A. L. Bleloch, K. S. Novoselov, A. Geim, and P. R. Briddon, Phys. Rev. B 77, 233406 (2008).

[50] P. Wachsmuth, R. Hambach, M. K. Kinyanjui, M. Guzzo, G. Benner, and U. Kaiser, Phys. Rev. B 88, 075433 (2013).

[51] P. Wachsmuth, R. Hambach, G. Benner, and U. Kaiser, Phys. Rev. B 90, 235434 (2014).

[52] E. A. Taft and H. R. Philipp, Phys. Rev. 138, A197 (1965).

[53] T. Ando, A. B. Fowler, and F. Stern, Rev. Mod. Phys. 54, 437 (1982).

[54] H. Yan, X. Li, B. Chandra, G. Tulevski, Y. Wu, M. Freitag, W. Zhu, P. Avouris, and F. Xia, Nat. Nanotechnol. 7, 330 (2012).

[55] M. Y. Han, B. Özyilmaz, Y. Zhang, and P. Kim, Phys. Rev. Lett. 98, 206805 (2007). 